\title{
Doğrusal Tamsayılı Programlama Problemlerinin Çözümü İçin Yeni Alternatif Bir Algoritma
}

\author{
Kadriye Şimşek Alan ${ }^{1 *}$ \\ 1*Y Yıldız Teknik Üniversitesi, Kimya-Metalurji Fakültesi, Matematik Mühendisliği Bölümü, İstanbul, Türkiye (ORCID: 0000-0001-6751-8013), ksimsek@yildiz.edu.tr
} (International Symposium on Multidisciplinary Studies and Innovative Technologies (ISMSIT) 2021 - 21-23 October 2021)

(DOI: 10.31590/ejosat.1019251)

\begin{abstract}
ATIF/REFERENCE: Şimşek Alan, K. (2021). Doğrusal Tamsayılı Programlama Problemlerinin Çözümü İçin Yeni Alternatif Bir Algoritma. Avrupa Bilim ve Teknoloji Dergisi, (29), 93-98.

\section{$\ddot{O} \mathbf{z}$}

Bu çalışmada doğrusal bir amaç fonksiyonuna ve doğrusal eşitlik veya eşitsizliklereden oluşan kısıtlara sahip olan Doğrusal Tamsayılı Programlama (DTP) Problemlerinin çözümü için yeni alternatif bir yöntem ve yeni alternatif bir algoritma sunulmaktadır. Yöntemimiz basit cebirsel işlemler ve matematik programlamaya dayanmaktadır. Doğrusal Tamsayılı Programlama Problemlerinin çözümünde kullanılan pek çok yöntem olmasına rağmen, bu yöntemlerin birçoğu uygulamada ve hesaplamada bazı güçlüklere sahiptir. Bu güçlüklere sahip olmayan yöntemimiz, diğer yöntemlere göre problemin sahip olduğu değişken sayısına daha az hassasdır. Bundan dolayı da çok sayıda değişkene sahip olan gerçek yaşam problemlerinin çözümünde de kullanılabilir. Ayrıca verilen problemin tüm alternatif çözümlerini de karar vericiye sunar. Önerilen yöntemin nasıl uygulandığını gösteren bir sayısal örnek verilerek Maple programlama dilinde kodlaması yapılmışıtır.
\end{abstract}

Anahtar Kelimeler: Doğrusal tamsayılı programlama, Doğrusal Diophantine denklemi, Optimum değer, Optimum çözüm, Optimal hiperdüzlem.

\section{A Novel Alternative Algorithm for Solving Linear Integer Programming Problems}

\begin{abstract}
In this study, a novel alternative method and a novel alternative algorithm are presented for the solution of Linear Integer Programming Problems that have a linear objective function and constraints consisting of linear equations or inequalities. Our method is based on simple algebraic operations and mathematical programming. Although there are many methods used in solving Linear Integer Programming Problems, most of these methods have some difficulties in application and computation. Our method, which does not have these difficulties, is easy to implement and less sensitive to the number of variables fo the problem than other methods. Therefore, it can also be used in solving real-life problems that have a large number of variables. It also presents all alternative solutions to the given problem to the decision maker. A numerical example showing how the proposed method is applied is given and coded in the Maple programming language.
\end{abstract}

Keywords: Integer linear programming, Linear Diophantine equations, Integer programming problems, Optimal solution.

\footnotetext{
* Sorumlu Yazar: ksimsek@yildiz.edu.tr
} 


\section{Giriş}

Doğrusal Programlama (DP), karar vericinin mevcut sınırlı kaynaklardan en iyi şekilde yararlanmak istediği üretim planlama ve karar verme gibi birçok alanda kullanılan matematiksel programlamanın bir alanıdır. Doğrusal programlama (DP) problemi, bir DP problemindeki karar değişkenlerinin kesirli değerler alabildiği, gerçek yaşam problemlerine en uygun çözümü verimli bir şekilde bulmayı amaçlar. Ancak gerçek hayattaki birçok uygulamada; karar değişkenlerinin kesirli değerlerinin kullanılması makul ve gerçekçi değildir. Bundan dolayı, gerçek yaşam problemlerinin çözümünde, Tamsayılı doğrusal programlama (TDP) kullanılır ve tamsayılı doğrusal programlama (TDP), yönetimsel kararları desteklemek için tamsayı değişkenleri içeren bir dizi doğrusal kısıtlamaya sahip olan doğrusal bir fonksiyonu optimize etmeyi amaçlar.

TDP problemleri, uygulama alanına bağlı olarak değişkenlerin bir kısmının veya tamamının tamsayı olması ile temsil edilebilir. Tamsayılı programlama "Kesikli optimizasyon" veya "Kombinatorik Optimizasyon" olarak da adlandırılır. Doğrusal programlama problemlerini çözmek için Simplex yöntemi geleneksel etkili bir yöntem olmasına ragmen TDP problemlerinin çözümünde henüz böyle etkili, kullanışlı bir yöntem bulunmuş değildir. İlk bakışta TDP problemlerinin çözümü DP problemlerinin çözümünden daha kolay görünmesine ragmen daha zordur ve tamsayılı programlama problemlerinin optimal çözümünü bulmak kolay değildir. Ancak buna rağmen yine de TDP bir çok araştırmacının ilgi odağı olmuştur ve bir çok bilim insanı bu alanda çalışmalar yapmıştır.

$\mathrm{Bu}$ araştırmacılardan biri olan Gomory, ilk olarak 1958'de DTP problemlerini çözmek için Gomory kesme düzlemi adı verilen bir yöntem geliştirdi (Gomory, 1958). Yöntem, bir DP probleminin çözümü ile başlar. Bulunan çözüm bir tamsayı ise optimal çözümdür. Değilse, çözümdeki kesirli değişkenler üzerinde oluşturulan doğrusal bir kısıt eklenerek tamsayılı bir çözüm aranır. DP problemlerinin çözümünde en çok tercih edilen yöntemlerden biri olan dal sınırı yöntemi ise 1960 yılında Land ve Doig tarafindan (Land ve Doig, 1960) tarafindan geliştirilmiştir.

Joseph, genel DTP probleminin çözümünde uç noktaların rolünü araştırdı ve uç noktaların optimal DTP çözümüne katkısını belirlemek için doğrusal bir programlama formülasyonu verdi ve ayrıca DTP'nin bir parametrik formülasyonunu da sundu (Joseph, 1995). Pandian ve Jayalakshmi ise DTP problemlerinin çözümü için, değişken indirgeme yöntemi olarak da adlandırılan bir yöntem yöntem geliştirmiştir (Pandian ve Jayalakshmi, 2012). Tsai ve arkadaşları genel bir DTP probleminin tüm çözümlerini bulmak için, küresel bir optimizasyon yaklaşımı önerdi ve ayrıca bir DTP problemine optimum çözümler bulmak için bir algoritma geliştirdi (Tsai ve ark., 2008). Muhammed ve Said saatlik gereksinim kalıpları ile bir genel personel çizelgeleme problemini aldı ve bunu bir doğrusal programlama problemi olarak formüle etti (Mohammed ve Said, 2013). DTP problemlerini çözmek için son 50 y1lda geliştirilen tekniklerin bir incelemesini ise Genova ve Guliashki yapmıştır (Genova ve Guliashki,2011). Hossain ve Hasan, sütun oluşturma yöntemine dayalı büyük ölçekli bir tamsayılı programlama problemini çözmek için bir algoritma geliştirmiş ve geliştirdikleri algoritmayı sermaye bütçelemesi ve planlamasını çözmek için kullanmışlardır (Hossain ve Hasan, 2013). Öte yandan Shinto ve Susmaha, Lineer Diophantine Denklemi kavramını kullanarak, Gevşek Doğrusal Programlamadan (GDP) elde edilen yaklaşık çözümün DTP için bir optimal bir çözüm olup olmadığını inceleyen bir test geliş̧irmişlerdir. Buna ek olarak, GDP'den elde edilen yaklaşık çözüm optimallik koşullarını sağlamasa bile DTP probleminin optimal çözümüne ulaşmak için dal-sınır yönteminin bir modifikasyonunu vermiştir (Shinto ve Susmaha, 2013). Bertsimas ve arkadaşları, TDP problemlerinin çözümü için cebirsel geometriye dayalı bir algoritma önermiş ve Farkas Lemma için doğal bir genelleme sağlamıştır (Bertsimas ve ark., 2000). Yöntem, duyarlılık analizi yapmak için doğal bir yol sağlar. Bu yol aynı zamanda tüm uygulanabilir çözümlerin sistematik siralamasını ve uygun tamsayılı programlama setinin yapısal bilgilerini verir. Tantawy, bir TDP problemini Gomory kesme düzlemi yöntemini kullanarak çözmek için eşlenik gradyan projeksiyon yöntemine dayalı yeni bir prosedür önerdi (Tantawy,2014). Dang ve Ye, tamsayılı programlama için "sabit nokta yinelemeli yöntem" olarak adlandırılan alternatif bir yöntem geliştirdi (Dang ve Ye, 2015). Pedrosa DTP problemlerini çözmek için, değişkenleri tamsayılı altkümeye ve sürekli altkümeye ayırma stratejisine dayanan evrimsel bir algoritma tanıttı (Pedrosa, 2002) Şimşek Alan ve arkadaşları, TDP problemlerinin çözümü için parametreleştirmeye dayalı, uygulaması kolay ve kullanışlı alternatif bir yöntem geliştirmiş ve iki değişkenli DTP problemlerinin çözümü için alternatif bir algoritma sunmuşlardır (Şimşek Alan ve ark., 2019). Şimşek Alan, sadece iki değişkenli DTP problemlerinin çözümü için uygulanan bu yöntemi üç değişkenli TDP problemlerinin çözümü için geliştirerek, kullanışlı alternatif bir algoritma sunmuştur (Şimşek Alan, 2020). Bilindiği gibi bir tamsayılı programlama probleminde değişken sayısı çok önemlidir. Değişken sayısı arttıkça problemin çözümündeki işlem yükü önemli ölçüde artar ve işlem süresi uzar. Bunun sonucunda da bilinen klasik yöntemlerin uygulanabilirliği düşer. $\mathrm{Bu}$ durum hem büyük boyutlu DT problemlerinin modellenmesinde hem de bu modellerin problem çözümünde kullanılmasında, etkin ve hızlı çalışan algoritmalar gereksinimini beraberinde getirmiştir.

$\mathrm{Bu}$ nedenle, bu çalışmada DTP problemlerini çözebilecek ve hesaplama zorluklarını aşabilecek bir yöntem önerilmiş ve kullanışlı alternatif bir algoritma sunulmuştur. Ayrıca önerilen yöntem verilen bir sayısal örneğe uygulanarak Maple programlama dilinde kodlanmıştır.

\section{Gerekli bilgiler}

Bu bölümde, gerekli kısa bilgiler sunulmaktadır.

Tanım 1: Bir DTP probleminin matematiksel formülasyonu aşağıda açıklanmıştır:

$$
P_{1}:\left\{\begin{array}{c}
\operatorname{Max}(\operatorname{Min}) \sum_{j=1}^{n} c_{j} x_{j} \\
\sum_{j=1}^{n} a_{i j} x_{j} \leq b_{i}, \\
x_{j} \geq 0 \text { ve tamsayl, } \quad(\mathrm{i}=1,2, \ldots \mathrm{m})
\end{array}\right.
$$

(Chen, 2011).

Tanım 2: Objektif hiper düzlemi düşünün $\sum c_{j} x_{j}=z$,

Burada $c_{j} \in Z$, tamsayılarda doğrusal bir Diophantine denklemidir. $\quad d=g d c\left(c_{j}, c_{j} \neq 0, j=1,2, \ldots, n\right)$ olsun. $\quad$ Bir Diophantine denklemi yalnızca ve ancak $\mathrm{d} \mid \mathrm{z}$ ise bir tamsayı çözümüne sahiptir. Ek olarak, doğrusal bir Diophantine 
denkleminin bir tamsayı çözümü varsa, bu denklem için sonsuz sayıda çözüm olacaktır (Schrijver, 1986)

Teorem 1: $\left(x_{1}, x_{2}, \ldots, x_{n}, z\right)$ problemin çözümüdür ancak ve ancak $\left(x_{1}, x_{2}, \ldots, x_{n}\right) P_{1}$ 'in tüm kısıtlamalarını sağlar (Schrijver, 1986).

\section{Tamsayılı Doğrusal Programlama Problemini Çözmek İçin Önerilen Algoritma}

\section{1. Çözüm Algoritmasının Adımları}

Çözüm algoritmamız aşağıdaki adımlardan oluşmaktadır.

Adım 0: $P_{1}$ problemini yükle.

Adım 1: Gevşetilmiş doğrusal programlama $P_{1}$ problemini çöz.

Adım 1' :Optimal çözüm tamsayı ise optimal çözüm bulunmuştur. Dur.aksi durumda 2. adıma git.

Adım 2: Optimal değeri $z^{\prime}$ in tamsayı kısmını $\sum_{j=1}^{n} c_{j} x_{j}$ ifadesine eşitleyerek $\sum_{j=1}^{n} c_{j} x_{j}=Z$ denkleminin oluştur.

Adım 3: Verilen DTP probleminin amaç fonksiyonunu ve kısıtlarını kullanarak herbir değişkenin tanım aralığını bul.

Adım 3': Bulunan tanım aralıkları anlamalı mı? Eğer anlamlıysa 4. adıma git. Anlamlı değilse optimal değer $z$ ' maksimum (minimum) probleminde bir birim düşür (arttır) ve 2. Adıma git.

Adım 4: Bulunan bu tanım aralıklarına göre hem oluşturulan Diophantine denklemini hem de kısttları sağlayan tamsayılı çözüm var mı? Eğer böyle tamsayılı çözümler varsa, optimum çözüm bulunmuştur, 5. Adıma git. Eğer yoksa optimal değer $z$ ' maksimum (minimum) probleminde bir birim düşür (arttır) ve 2 . Adıma git.

Adım 5: Hem verilen DTP problemlerini sağlayan hem de de kısıtları sğlayan tamsayılı çözümleri yaz.

Adım 6: Dur. 


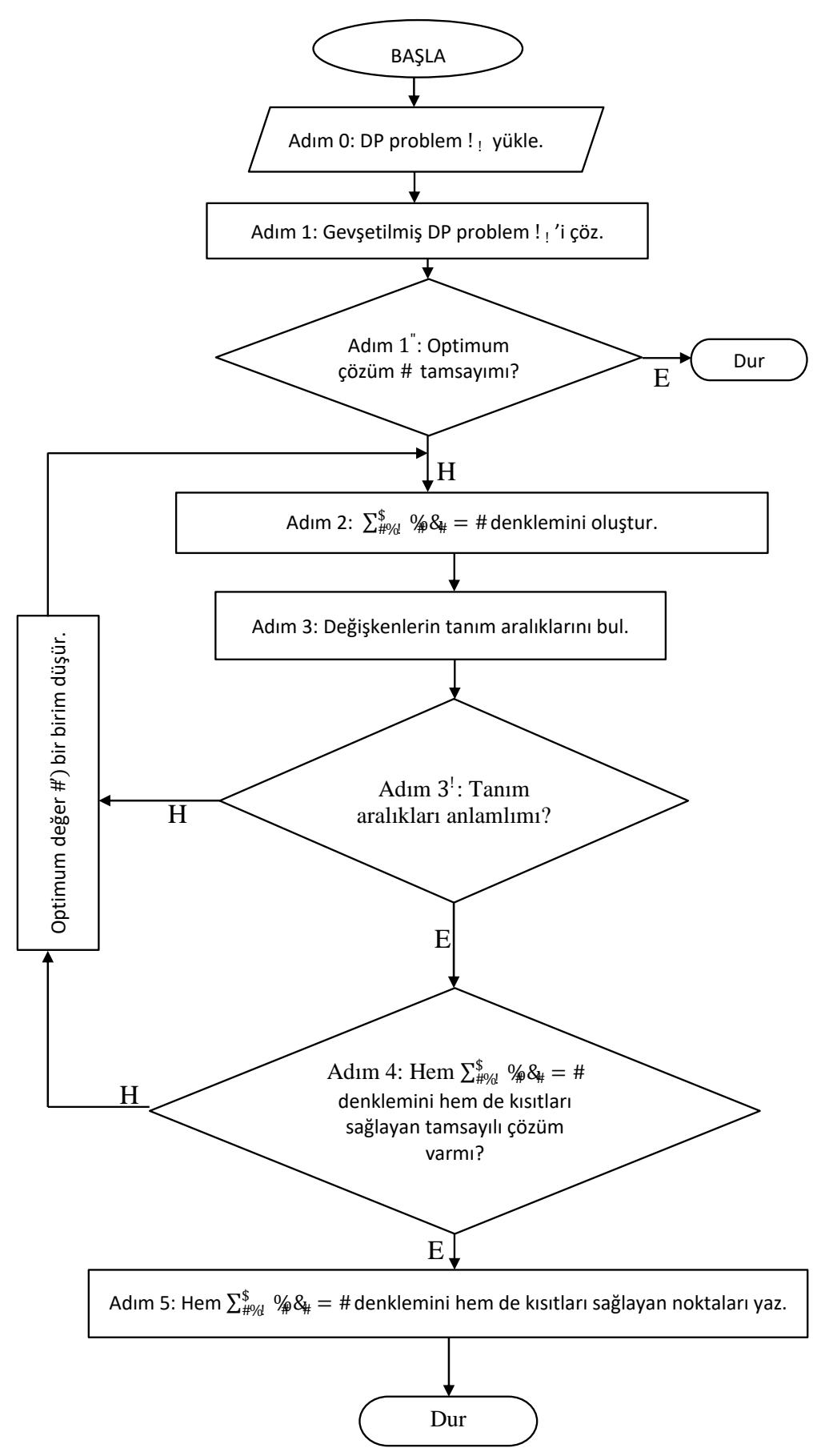

Şekil 1: Önerilen çözüm yönteminin algoritması 


\subsection{Sayısal Örnek}

Örnek 1. Aşağıda verile DTP problemini çözelim.

$$
\begin{gathered}
\text { Maks. } z=3 x_{1}+2 x_{2}-5 x_{3}-2 x_{4}+3 x_{5} \\
x_{1}+x_{2}+x_{3}+2 x_{4}+3 x_{5} \leq 4 \\
7 x_{1}+3 x_{3}+2 x_{4}+x_{5} \leq 8 \\
11 x_{1}-6 x_{2}+3 x_{3}-3 x_{5} \geq 4 \\
x_{1} \geq 0, x_{2} \geq 0, x_{3} \geq 0, x_{4} \geq 0, x_{5} \geq 0 \text { ve tamsay } 1
\end{gathered}
$$

Adım 0: Maks. $z=3 x_{1}+2 x_{2}-5 x_{3}-2 x_{4}+3 x_{5}$

$$
\begin{gathered}
x_{1}+x_{2}+x_{3}+2 x_{4}+3 x_{5} \leq 4 \\
7 x_{1}+3 x_{3}+2 x_{4}+x_{5} \leq 8 \\
11 x_{1}-6 x_{2}+3 x_{3}-3 x_{5} \geq 4
\end{gathered}
$$

$x_{1} \geq 0, x_{2} \geq 0, x_{3} \geq 0, x_{4} \geq 0, x_{5} \geq 0$ ve tamsay1.

Adım 1: Gevşetilmiş DP problem $P_{1}$ çözülürse, optimal çözüm $P_{1}:\left(x_{1}, x_{2}, x_{3}, x_{4}, x_{5}\right)=(1.0598,1.1364,0,0,0.623)$ ve optimal değer $z=7.25$ bulunur.

Adım 2: $3 x_{1}+2 x_{2}-5 x_{3}-2 x_{4}+3 x_{5}$ ifadesi 7 değerine eşitlenirse $3 x_{1}+2 x_{2}-5 x_{3}-2 x_{4}+3 x_{5}=7$

Diophantine denklemi elde edilir.

Adım 3: Kısıtlardan, değişkenlerin tanım aralıkları $0 \leq x_{1} \leq$ $1,0 \leq x_{2} \leq 4,0 \leq x_{3} \leq 2,0 \leq x_{4} \leq 2,0 \leq x_{5} \leq 1 \quad$ olarak elde edilir.

Adım 4: $3 x_{1}+2 x_{2}-5 x_{3}-2 x_{4}+3 x_{5}=7 \quad$ Diophantine denklemini sağlayan tamsayılı çözüm yok. Bu nedenle optimal değer 7, bir birim düşürülürek 6 alınır ve 2 . Adıma gidilir. Daha sonra $x_{1}+2 x_{2}-5 x_{3}-2 x_{4}+3 x_{5}=6$ denklemi oluşturulur. Verilen algoritmanın adımları uygulanırsa, bu denklemini de sağlayan tamsayılı çözüm olmadığı görülür. Bundan dolayı, optimal değer, bir birim daha düşürülür ve 2 . Adıma gidilir ve $x_{1}+2 x_{2}-5 x_{3}-2 x_{4}+3 x_{5}=5$ denklemi oluşturulur.

Adım 5: Değişkenlerin tanım aralıklarına göre, hem $x_{1}+2 x_{2}-$ $5 x_{3}-2 x_{4}+3 x_{5}=5$ Diophantine denklemini hem de kısitları sağlayan tamsayılı nokta $(1,1,0,0,0)$ noktasıdır.

Adım 6: Dur.

\section{3. Örnek 1'in Maple Programlama Dilinde Kodu}

Örnek 4.1'in Maple programlama dilindeki kodlaması aşağıdaki gibidir.

$>$ \#print ("a=", 7-s);

for $x 1$ from 0 to 1 do;

for $x 2$ from 0 to 4 do;

for $x 3$ from 0 to $2 t$ do;

for $x 4$ from 0 to 2 do;

for $x 5$ from 0 to $1 \mathrm{do}$;

$M:=3 * x 1+2 * \times 2-5 * x 3-2 * x 4+3 * \times 5$

d1: $=x 1+x 2+x 3+2 * x 4+x 5$;

d2: $=7 * \times 1+3 * \times 3-4 * \times 4+3 * \times 5$;

d3: $=11 * \times 1-6 * \times 2+3 * \times 4-3 * \times 5$; aa:=0;

if $((M=7-s)$ and $(\mathrm{d} 1<=4)$ and $(\mathrm{d} 2<=8)$ and $(\mathrm{d} 3>=3))$ then

print ( "Maksimum Deger", 7-s, "Optimum çözüm"

$(\mathrm{x} 1, \mathrm{x} 2, \mathrm{x} 3, \mathrm{x} 4, \mathrm{x} 5))$

else end if;

end do; end do;

end do;

end do;

end do;

end do;

$$
a a:=1
$$

"Maksimum Deðer", 5, "Optimum çözüm" (1, 1, 0, 0, 0 )

\section{Sonuç}

Bu çalışmada, DTP problemlerinin çözümü için yeni iterativ bir yöntem geliştirilmiş ve kolay uygulanabilir alternatif bir algoritma sunulmuştur. Bu çalışmada önerilen yöntemimizin DTP problemlerinin çözümünde kullanılan diğer yöntemler ile karşılaştırıldığında, diğer yöntemlere göre birçok avantajı vardır ve bu avantajları aşağıdaki gibi ifade edilebilir.

1. DTP problemleri, hesaplama ve işlem süresi açısından karmaşık problemlerdir. Yöntemimiz diğer yöntemlere göre daha kolay uygulanabilirdir ve daha kısa sürede sonuca ulaşır.

2. Tamsayılı programlamada hesaplamaları etkileyen en önemli faktör değişken sayısıdır. Grafik Yöntemi sadece iki değişkenli DTP problemlerine uygulanabilir. Dal-sınırı ve düzlem kesme algoritmaları gibi yöntemler değişken sayısı arttıkça kullanışlılığını ve uygulanabilirliğini kaybetmektedir. Yöntemimiz, bu yöntemlere göre değişken sayısına daha az hassastır ve daha fazla değişken içeren DTP problemlerinin çözümünde kullanılabilir.

3. Dal-sınır ve yuvarlama gibi yöntemlerde olası optimal çözümler göz ardı edilebilir, ancak bizim yöntemimizde hiçbir optimal çözüm göz ardı edilmez. Ayrıca problemin tüm alternatif çözümleri bulunarak, karar vericiye sunulur.

4. Bizim yöntemimiz de hiçbir yeni kısıt eklenmez.

\section{Teşekkür}

Bu çalışma Yıldız Teknik Üniversitesi Bilimsel Araştırma Projeleri Koordinasyon Birimi tarafından desteklenmiştir. Proje Numaras1: FBA-2021-4032

\section{Kaynakça}

Bertsimas, D., Perakis, G., Tayur, S. (2000). A new algebraic geometry algorithm for integer programming. Management Science, 46(7), 999-1008.

Chen, D. S., Batson, R. G., Dang, Y. (2015). Applied integer programming: modeling and solution, pp. 3-4. John Wiley \& Sons, New Jersey, 2011.

Dang, C., Y. Ye. (2015). A fixed point iterative approach to integer programming and its distributed computation. - Fixed Point Theory and Applications. 182, 1-15.

Genova, K., Guliashki, V. (2011). Linear integer programming methods and approaches-a survey. - Journal of Cybernetics and Information Technologies, 11(1), 1-23.

Gomory, Ralph E. (1958) Outline of an Algorithm for Integer Solutions to Linear Programs. Bull. Amer. Math Soc. 64(5): 275-278.

Hossain, M. I., Hasan, M. B. (2013). A Decomposition Technique For Solving Integer Programming Problems. GANIT: Journal of Bangladesh Mathematical Society, 33, 1-11. 
Joseph, A. (1995). Parametric formulation of the general integer linear programming problem. - Computers \& operations research, 22(3), 883-892.

Mohamad, N. H., \& Said, F. (2013). Integer linear programming approach to scheduling toll booth collectors problem. Indian Journal of Science and Technology, 6(5), 4416-4421.

Pandian, P., \& Jayalakshmi, M.(2012). A New Approach for solving a Class of Pure Integer Linear Programming Problems. Journal of Advanced Engineering Technology, 3, 248-251.

Pedroso, J. P. (2002). An evolutionary solver for pure integer linear programming. International Transactions in Operational Research, 9(3), 337-352.

Schrijver, A.(1986). "Theory of Linear and Integer Programming", John Wiley \& Sons Ltd.

Shinto, K. G., \& Sushama, C.M. (2013). An Algorithm for Solving Integer Linear Programming Problems. International Journal of Research in Engineering and Technology, 37-47.

Simsek Alan, K., Albayrak, I., M., Sivri, M., Guler, C. (2019). An Alternative Algorithm for Solving Linear Programming Problems Having Two Variables, - International Journal of Applied Information Systems. 12 (25), 6-9.

Simsek Alan, K. (2020). An Novel Algorithm for Solving Linear Programming Problems Having Three Variables. J. Cyber. and Inform. Technologies 20 (4), 27-35.

Tantawy, S. F. (2014). A new procedure for solving integer linear programming problems. - Arabian Journal for Science and Engineering. 39 (6), 5265-5269.

Tsai, J. F., Lin, M. H., Hu, Y. C. (2008). Finding multiple solutions to general integer linear programs. - European Journal of Operational Research, 184(2), 802-809. 